\title{
On the reflected wave superposition method for a travelling string with mixed boundary supports
}

\author{
E.W. Chen ${ }^{\mathrm{a}, *}$, K. Zhang ${ }^{\mathrm{a}}$, N.S. Ferguson ${ }^{\mathrm{b}}$, J. Wanga ${ }^{\mathrm{a}}$, Y.M. Lu ${ }^{\mathrm{a}}$
}

aschool of Mechanical Engineering, Hefei University of Technology, Hefei, 230009, China

bInstitute of Sound and Vibration Research, University of Southampton, Southampton SO171BJ, England, UK

\begin{abstract}
An analytical vibration response in the time domain for an axially translating and laterally vibrating string with mixed boundary conditions is considered in this paper. The domain of the string is a constant, dependent upon the general initial conditions. The translating tensioned strings possess different types of mixed boundary conditions, such as fixed dashpot, fixed spring-dashpot, fixed mass-spring-dashpot. An analytical solution using a reflected wave superposition method is presented for a finite translating string. Firstly, the cycle of boundary reflection for strings is provided, which is dependent upon the string length. Each cycle is divided into three time intervals according to the travelling speed and direction of the string. Applying D'Alembert's principle and the reflection properties, expressions for the reflected waves under three different non-classical boundary conditions are derived. Then, the vibrational response of the axially translating string is solved for three time intervals by using a reflected wave superposition method. The accuracy and efficiency of the proposed method are confirmed numerically by comparison to simulations produced using a Newmark- $\beta$ method solution. The energy expressions for a travelling string with a fixed dashpot boundary condition is obtained and the time domain curves for the total energy and the change of energy at the boundaries are given.
\end{abstract}

Keywords: translating string; mixed boundaries; boundary reflection; D 'Alembert principle; propagating wave

\section{Introduction}

In the present study, a uniform finite length string model with uniform density $\rho$ is considered, which is travelling over two smooth supports under constant speed $v$ and constant tension $T$. The concept of wave propagation is used in this model, where the reflections of waves occur at both boundaries, which represents many real engineering slender systems, such as conveyor belts, elevator cables, power transmission belts and 
magnetic tapes. The phenomena of propagation and reflection of waves in one-dimensional wave bearing systems, such as strings, have been studied for many years, it is though still of great research interest due to their theoretical importance and application.

A large number of examples have been studied on the reflection phenomena, such as, the classical D'Alembert principle was used to study the reflection phenomenon in either an infinite or a semi-infinite stationary string with classical boundary conditions[1,2]. Akkaya, Gaiko and Van Horssen [3,4] obtained the exact free, linear, lateral vibration of both a stationary [3] and an axially travelling [4] semi-infinite string applying the same method. Various alternative approaches have been applied to solve and obtain the response of axially moving materials. Yang and Tan [5] studied both a travelling string and beam using a transfer function method, which for the latter considered a damped, axially moving beam over a set of different boundary conditions. Based on the transfer function formulation and wave propagation, Tan and Ying [6] subsequently derived an exact solution for the response of a translating string with general boundary conditions. Van Horssen [7] used a Laplace transform method instead, constructing exact solutions of the lateral vibrations in travelling strings due to small lateral vibrations of the supports. Miranker[8] studied a constant length model for the transverse vibrations of a tape moving between a pair of pulleys, which is a similar type of problem as considered in this paper. The solution [8] comprises two travelling waves or by a modal description and includes reflection from the classical fixed-fixed boundaries, but not the reflection of non-classical boundaries. Lee [9] analyzed free vibration of a string with time-varying length, by dealing with travelling waves and obtained an exact solution.

Simple models which describe these vibrations can be expressed as initial-boundary value problems. Darmawijoyo [10,11] studied such an initial-boundary value problem with a non-classical boundary condition, constructing asymptotic approximations of the solution for an axially travelling string by a multiple-timescales perturbation method. Chen and Ferguson [12] more recently studied the lateral vibration and the energy dissipation in a travelling string attached to a viscous damper at one end, using a time varying state space function method and the Newmark- $\beta$ method. In terms of dissipative boundaries, Gaiko and Van Horssen [13] also gave a complete and accurate description of the damping and the low frequency oscillatory behaviour of the travelling string with an attached spring-mass-dashpot system at one end. Chen's previous study [14] considered a reflected wave superposition method for vibration and energy of a travelling string. In that study, the translating tensioned strings possess either fixed-fixed or fixed-free boundaries. Furthermore, vibration control of a fixed-length string system have been studied extensively, and the study of the variable length string was extended. 
Chang [15] and Ngo [16] investigated an active vibration control and an adaptive boundary control at one end of the axially travelling string modelled based on the Hamilton's principle.

Previous work on a travelling string defined on a finite domain covers both classical boundaries or a semi-finite domain with non-classical boundary, In contrast, more recently the classical D'Alembert formula in conjunction with the characteristic coordinates method was used to solve an initial value problem for a wave equation on a bounded interval with a Dirichlet type of boundary condition at one end and a Robin boundary condition at the other [17]. The present work focuses on the analytical free lateral vibration of an axially translating string with mixed boundary conditions and constant length. The solution of the vibration response with the mixed boundary conditions and the multiple reflections will exist in a finite domain. A reflected wave superposition method is proposed and completely developed in this study. At both ends of the axially travelling string the multiple reflections of the propagating waves are studied. The expressions for the reflected waves at the two boundaries are derived. Superposition of all of the incident and reflected waves provides results for the free vibration of the string over the three time intervals. This work provides an analytical methodology to solve the translating media problem defined over a finite domain with different mixed boundary conditions and the details of the process are given.

This paper is organized as follows. As a first step, the governing equations for the lateral vibration of an axially travelling finite string with the relevant boundary conditions are derived using the extended form of Hamilton's principle, and the dimensionless problem is given in Section 2. Section 3 shows the derivation of the formula for the reflected wave at both ends of a mixed boundary. In Section 4, the time varying cycle is investigated. Next, the reflected wave superposition method is used to obtain the response due to the initial conditions of a travelling string between two types of boundaries separated by a constant distance, i.e. the length of string between the boundaries is also constant. In section 5 , the fixed dashpot (viscous damper) boundary condition is considered as an example for which the vibrational energy is obtained using the proposed reflected wave superposition method. Finally, Section 6 provides the main conclusions.

\section{Establishment of motion model of travelling string system}

The model of an axially travelling string system with classical and non-classical mixed boundaries is shown in Fig.1, where $\rho$ is the uniform string mass per unit length, $T$ is the uniform tension, $v$ is the assumed constant translational speed of the string and $l_{0}$ is the length of the string. The viscous damping coefficient at the right boundary is $\eta$, the stiffness of the spring is $k$ and the mass at the right boundary in Fig.1(c) is $m . u(x, t)$ represents the transverse displacement of the axial moving string at the coordinate $x$ and the time $t$. To avoid the divergence instability [18] in the string and to allow any propagating wave in the string direction to be reflected at the other end, the string translational speed $v$ is assumed to be less than the free wave propagation speed $c$, i.e . 
$|v|<c$.

In addition, this model is based on the following assumptions: (1) In the process of movement, the density, cross sectional area, elastic modulus and tension of the string remain constant. (2) The model neglects the effect of longitudinal vibration of the moving string. (3) The effect of various frictional forces on the system is also neglected.

The equation of motion for the lateral vibration of a travelling string between two boundaries can be obtained by Hamilton's Principle $[13,19,20]$ and is given by

$$
u_{t t}+2 v u_{x t}+\left(v^{2}-c^{2}\right) u_{x x}=0
$$

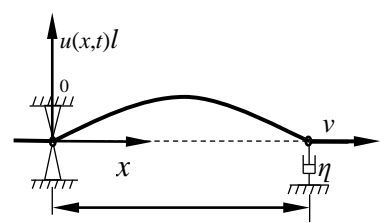

(a)

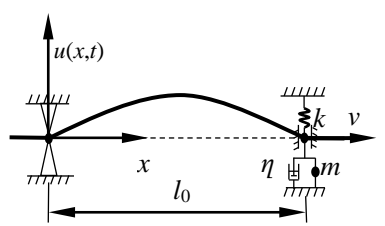

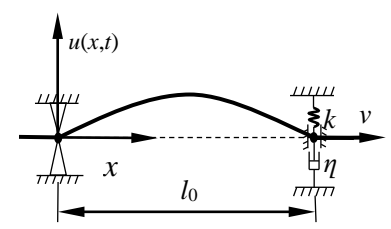

(b)

(c)

Fig.1 Simplified model of classical and non-classical mixed boundary of axially moving string. (a) is fixed dashpot boundary, (b) is fixed spring-dashpot boundary and (c) is fixed mass-spring-dashpot boundary.

The corresponding boundary equations for the axial moving string model shown respectively in Fig.1 (a), (b) and (c) are given by [4,13]

$$
\begin{gathered}
\left\{\begin{array}{c}
u(0, t)=0 \\
\eta u_{t}\left(l_{0}, t\right)=\rho v\left[u_{t}\left(l_{0}, t\right)+v u_{x}\left(l_{0}, t\right)\right]-T u_{x}\left(l_{0}, t\right)
\end{array}\right. \\
\left\{\begin{array}{c}
u(0, t)=0 \\
k u\left(l_{0}, t\right)+\eta u_{t}\left(l_{0}, t\right)=\rho v\left[u_{t}\left(l_{0}, t\right)+v u_{x}\left(l_{0}, t\right)\right]-T u_{x}\left(l_{0}, t\right)
\end{array}\right. \\
\left\{\begin{array}{c}
u(0, t)=0 \\
m u_{t t}\left(l_{0}, t\right)+k u\left(l_{0}, t\right)+\eta u_{t}\left(l_{0}, t\right)-\rho v u_{t}\left(l_{0}, t\right)-\left(\rho v^{2}-T\right) u_{x}\left(l_{0}, t\right)=0
\end{array}\right.
\end{gathered}
$$

\section{The reflection law for waves incident upon the mixed boundary condition}


The general one-dimensional wave solution of Eq. (1) for the string displacement using the D'Alembert method is well known ${ }^{[2,21]}$. It is given by

$$
u(x, t)=F\left(x-v_{r} t\right)+G\left(x+v_{l} t\right)
$$

Here, $F\left(x-v_{r} t\right)$ is the right-propagating wave with speed of $v_{r}=c+v$ and $G\left(x+v_{l} t\right)$ is the left-propagating wave with speed of $v_{l}=c-v$. The initial conditions for the string vibration are assumed as follows

$$
\left\{\begin{array}{l}
u(x, 0)=\phi(x) \\
u_{t}(x, 0)=\psi(x)
\end{array}, 0 \leq x \leq l_{0}\right.
$$

Substituting Eq. (5) into Eq. (6),one has the expressions for $F(x)$ and $G(x)$ given by

$$
\left\{\begin{array}{l}
F(x)=\frac{v_{l}}{v_{r}+v_{l}} \phi(x)+\frac{1}{v_{r}+v_{l}} \int_{x}^{l_{0}} \psi(\xi) \mathrm{d} \xi+C \\
G(x)=\frac{v_{r}}{v_{r}+v_{l}} \phi(x)-\frac{1}{v_{r}+v_{l}} \int_{x}^{l_{0}} \psi(\xi) \mathrm{d} \xi-C
\end{array}\right.
$$

where, $C$ is an integral constant.

\subsection{Fixed dashpot boundary reflection}

According to Fig.1 (a), substituting the general solution in Eq. (5) into the boundary conditions in Eq. (2), one can obtain

$$
\left\{\begin{array}{c}
F\left(-v_{r} t\right)=-G\left(v_{l} t\right) \\
G^{\prime}\left(l_{0}+v_{l} t\right)=\beta F^{\prime}\left(l_{0}-v_{r} t\right)
\end{array}\right.
$$

where,

$$
\beta=\frac{-\eta v_{r}+\rho v c+T}{-\eta v_{l}+\rho v c-T}
$$

Defining $s=l_{0}+v_{l} t>0$, the second equation in Eq. (8) can be transformed into

$$
G^{\prime}(s)=\beta F^{\prime}\left(l_{0}+\frac{v_{r}}{v_{l}} l_{0}-\frac{v_{r}}{v_{l}} s\right)
$$

The integration of Eq. (9) gives the following equation

$$
\int_{x}^{l_{0}} G^{\prime}(\mathrm{s}) \mathrm{d} s=\int_{x}^{l_{0}} \beta F^{\prime}\left(l_{0}+\frac{v_{r}}{v_{l}} l_{0}-\frac{v_{r}}{v_{l}} s\right) \mathrm{d} s
$$

Then the solution of $G(x)$ can be obtained

$$
G(x)=G\left(l_{0}\right)+\beta \frac{v_{l}}{v_{r}} F\left(l_{0}\right)-\beta \frac{v_{l}}{v_{r}} F\left(\frac{2 c}{v_{l}} l_{0}-\frac{v_{r}}{v_{l}} x\right)
$$

Combining Eq.(8) and Eq.(11), one has the equation for the boundary reflection with the 
fixed dashpot boundary condition:

$$
\left\{\begin{array}{l}
F_{r}(x)=-G_{i}\left(-\frac{v_{l}}{v_{r}} x\right) \\
G_{r}(x)=G_{r}\left(l_{0}\right)+\beta \frac{v_{l}}{v_{r}} F_{i}\left(l_{0}\right)-\beta \frac{v_{l}}{v_{r}} F_{i}\left(\frac{2 c}{v_{l}} l_{0}-\frac{v_{r}}{v_{l}} x\right)
\end{array}\right.
$$

where, $F_{i}, G_{i}$ represent the incident waves $(i=1,2,3, \ldots)$ and $F_{r}, G_{r}$ represent the reflected waves $(r=i+1)$.

\subsection{Fixed spring-dashpot boundary reflection}

According to Fig.1 (b) and substituting Eq. (5) into the boundary condition of Eq. (3), one can obtain Eq. (13) and Eq. (14) as follows

$$
\begin{gathered}
F\left(-v_{r} t\right)=-G\left(v_{l} t\right) \\
k\left[F\left(l_{0}-v_{r} t\right)+G\left(l_{0}+v_{l} t\right)\right]+\eta\left[-v_{r} F^{\prime}\left(l_{0}-v_{r} t\right)+v_{l} G^{\prime}\left(l_{0}+v_{l} t\right)\right]= \\
\rho v\left[-v_{r} F^{\prime}\left(l_{0}-v_{r} t\right)+v_{l} G^{\prime}\left(l_{0}+v_{l} t\right)+v F^{\prime}\left(l_{0}-v_{r} t\right)+v G^{\prime}\left(l_{0}+v_{l} t\right)\right] \\
-T\left[F^{\prime}\left(l_{0}-v_{r} t\right)+G^{\prime}\left(l_{0}+v_{l} t\right)\right]
\end{gathered}
$$

Introducing the following notations for convenience into Eq. (14)

$$
\left\{\begin{array}{l}
\alpha=\frac{k}{\rho v c-\eta v_{l}-T} \\
\beta=\frac{\rho v c-\eta v_{r}+T}{\rho v c-\eta v_{l}-T}
\end{array}\right.
$$

one has

$$
G^{\prime}\left(l_{0}+v_{l} t\right)-\alpha G\left(l_{0}+v_{l} t\right)=\beta F^{\prime}\left(l_{0}-v_{r} t\right)+\alpha F\left(l_{0}-v_{r} t\right)
$$

Defining $s=l_{0}+v_{l} t>0$, the above form can be converted to:

$$
G^{\prime}(s)-\alpha G(s)=\beta F^{\prime}\left(l_{0}+\frac{v_{r}}{v_{l}} l_{0}-\frac{v_{r}}{v_{l}} s\right)+\alpha F\left(l_{0}+\frac{v_{r}}{v_{l}} l_{0}-\frac{v_{r}}{v_{l}} s\right)
$$

Using the integrating factor $\mathrm{e}^{-\alpha s}$, the integration for Eq. (17) yields:

$$
\int_{x}^{l_{0}}\left[G^{\prime}(\mathrm{s})-\alpha G(s)\right] \mathrm{e}^{-\alpha s} \mathrm{~d} s=\int_{x}^{l_{0}}\left[\beta F^{\prime}\left(\frac{2 c}{v_{l}} l_{0}-\frac{v_{r}}{v_{l}} s\right)+\alpha F\left(\frac{2 c}{v_{l}} l_{0}-\frac{v_{r}}{v_{l}} s\right)\right] \mathrm{e}^{-\alpha s} \mathrm{~d} s
$$

Hence, we have the solution:

$$
\begin{aligned}
G(x)= & \left(G\left(l_{0}\right)+\beta \frac{v_{l}}{v_{r}} F\left(l_{0}\right)\right) \mathrm{e}^{\alpha\left(x-l_{0}\right)}-\beta \frac{v_{l}}{v_{r}} F\left(\frac{2 c}{v_{l}} l_{0}-\frac{v_{r}}{v_{l}} x\right)+ \\
& \alpha\left(\beta \frac{v_{l}}{v_{r}}-1\right) \int_{x}^{l_{0}} F\left(\frac{2 c}{v_{l}} l_{0}-\frac{v_{r}}{v_{l}} s\right) \mathrm{e}^{\alpha(x-s)} \mathrm{d} s
\end{aligned}
$$

As a final step, combining Eq.(13) and Eq.(19), one has the equation for the boundary 
reflection with the fixed spring-dashpot boundary condition:

$$
\left\{\begin{aligned}
F_{r}(x)= & -G_{i}\left(-\frac{v_{l}}{v_{r}} x\right) \\
G_{r}(x)= & \left(G_{r}\left(l_{0}\right)+\beta \frac{v_{l}}{v_{r}} F_{i}\left(l_{0}\right)\right) \mathrm{e}^{\alpha\left(x-l_{0}\right)}-\beta \frac{v_{l}}{v_{r}} F_{i}\left(\frac{2 c}{v_{l}} l_{0}-\frac{v_{r}}{v_{l}} x\right)+ \\
& \alpha\left(\beta \frac{v_{l}}{v_{r}}-1\right) \int_{x}^{l_{0}} F_{i}\left(\frac{2 c}{v_{l}} l_{0}-\frac{v_{r}}{v_{l}} s\right) \mathrm{e}^{\alpha(x-s)} \mathrm{d} s
\end{aligned}\right.
$$

where, $F_{i}, G_{i}$ represent the incident waves $(i=1,2,3, \ldots)$ and $F_{r}, G_{r}$ represent the reflection waves $(r=i+1)$.

\subsection{Fixed mass-spring-dashpot boundary reflection}

According to Fig.1 (c) and substituting Eq.(5) into the boundary conditions of Eq. (4) and Eq. (13) one can obtain the expressions as follows:

$$
\begin{aligned}
& F\left(-v_{r} t\right)=-G\left(v_{l} t\right) \\
& m\left[v_{r}^{2} F^{\prime \prime}\left(l_{0}-v_{r} t\right)+v_{l}^{2} G^{\prime \prime}\left(l_{0}+v_{l} t\right)\right]+k\left[F\left(l_{0}-v_{r} t\right)+G\left(l_{0}+v_{l} t\right)\right] \\
& +\eta\left[-v_{r} F^{\prime}\left(l_{0}-v_{r} t\right)+v_{l} G^{\prime}\left(l_{0}+v_{l} t\right)\right] \\
& =\rho v\left[-v_{r} F^{\prime}\left(l_{0}-v_{r} t\right)+v_{l} G^{\prime}\left(l_{0}+v_{l} t\right)+v F^{\prime}\left(l_{0}-v_{r} t\right)+v G^{\prime}\left(l_{0}+v_{l} t\right)\right] \\
& -T\left[F^{\prime}\left(l_{0}-v_{r} t\right)+G^{\prime}\left(l_{0}+v_{l} t\right)\right]
\end{aligned}
$$

In order to simplify Eq. (22), the following symbols are introduced:

$$
\left\{\begin{array}{l}
s=l_{0}+v_{l} t \\
\alpha^{2}=\frac{k}{m v_{l}^{2}} \\
\beta_{0}^{2}=\frac{\left(\eta v_{l}-\rho v c+T\right)^{2}}{4 m k v_{l}^{2}}
\end{array}\right.
$$

Substituting Eq. (23) into Eq. (22),one has

$$
G^{\prime \prime}(s)+2 \beta_{0} \alpha G^{\prime}(s)+\alpha^{2} G(s)=R\left(\frac{2 c l_{0}}{v_{l}}-\frac{v_{r}}{v_{l}} s\right)
$$

where,

$$
\begin{aligned}
& R\left(\frac{2 c l_{0}}{v_{l}}-\frac{v_{r}}{v_{l}} s\right)=-\frac{v_{r}^{2}}{v_{l}^{2}} F^{\prime \prime}\left(\frac{2 c l_{0}}{v_{l}}-\frac{v_{r}}{v_{l}} s\right)+\frac{\eta v_{r}-\rho v c-T}{m v_{l}^{2}} F^{\prime}\left(\frac{2 c l_{0}}{v_{l}}-\frac{v_{r}}{v_{l}} s\right) \\
& -\frac{k}{m v_{l}^{2}} F\left(\frac{2 c l_{0}}{v_{l}}-\frac{v_{r}}{v_{l}} s\right)
\end{aligned}
$$


In combination of Eq. (24) with Eq. (7), one knows that the function $G(s)$ in Eq. (24) satisfies the following expressions:

$$
\left\{\begin{array}{l}
G\left(l_{0}\right)=\frac{v_{r}}{v_{r}+v_{l}} \phi\left(l_{0}\right) \\
G^{\prime}\left(l_{0}\right)=\frac{v_{r}}{v_{r}+v_{l}} \phi^{\prime}\left(l_{0}\right)+\frac{1}{v_{r}+v_{l}} \psi\left(l_{0}\right)
\end{array}\right.
$$

For convenience, one sets the integral constant $C=0$ in the following calculations. The method of variation of parameters [2,3] is used for solving the second order differential equation (24). The corresponding characteristic equation for Eq. (24) is given by

$$
\mu^{2}+2 \alpha \beta_{0} \mu+\alpha^{2}=0
$$

where three cases follow as

$$
\mu= \begin{cases}\alpha\left(-\beta_{0} \pm \sqrt{\beta_{0}^{2}-1}\right), & \beta_{0}>1 \\ -\alpha \beta_{0}, & \beta_{0}=1 \\ \alpha\left(-\beta_{0} \pm i \sqrt{1-\beta_{0}^{2}}\right), & \beta_{0}<1\end{cases}
$$

corresponding to overdamped, critically damped, and underdamped system, respectively.

\subsubsection{The overdamped case $\left(\beta_{0}>1\right)$}

For the case of the damping ratio $\beta_{0}>1$, the roots of Eq. (27) are given by:

$$
\left\{\begin{array}{l}
\mu_{1}=\alpha\left(-\beta_{0}+\sqrt{\beta_{0}^{2}-1}\right) \\
\mu_{2}=\alpha\left(-\beta_{0}-\sqrt{\beta_{0}^{2}-1}\right)
\end{array}\right.
$$

By using these roots and the method of variation of parameters, one obtains the general solution for $G(s)$ as follows:

$$
\begin{aligned}
& G(s)=C_{1} \mathrm{e}^{\mu_{1} s}+C_{2} \mathrm{e}^{\mu_{2} s} \\
& +\mathrm{e}^{\mu_{1} s} \int_{s}^{l_{0}}\left[\mathrm{e}^{\left(\mu_{2}-\mu_{1}\right) s} \int_{s}^{l_{0}} R\left(\frac{2 c l_{0}}{v_{l}}-\frac{v_{r}}{v_{l}} x\right) \mathrm{e}^{-\mu_{2} x} \mathrm{~d} x\right] \mathrm{ds}=C_{1} \mathrm{e}^{\mu_{1} s}+C_{2} \mathrm{e}^{\mu_{2} s} \\
& +\frac{1}{\mu_{2}-\mu_{1}}\left[\mathrm{e}^{\mu_{2} s} \int_{s}^{l_{0}} R\left(\frac{2 c l_{0}}{v_{l}}-\frac{v_{r}}{v_{l}} x\right) \mathrm{e}^{-\mu_{2} x} \mathrm{~d} x-\mathrm{e}^{\mu_{1} s} \int_{s}^{l_{0}} R\left(\frac{2 c l_{0}}{v_{l}}-\frac{v_{r}}{v_{l}} x\right) \mathrm{e}^{-\mu_{1} x} \mathrm{dx}\right]
\end{aligned}
$$

The parameters of $C_{1}$ and $C_{2}$ can be obtained through Eq. (26): 


$$
\left\{\begin{array}{l}
C_{1}=\frac{-v_{r} \phi^{\prime}\left(l_{0}\right)+\mu_{2} v_{r} \phi\left(l_{0}\right)-\psi\left(l_{0}\right)}{\left(v_{r}+v_{l}\right)\left(\mu_{2}-\mu_{1}\right) \mathrm{e}^{\mu_{1} l_{0}}} \\
C_{2}=\frac{v_{r} \phi^{\prime}\left(l_{0}\right)-\mu_{1} v_{r} \phi\left(l_{0}\right)+\psi\left(l_{0}\right)}{\left(v_{r}+v_{l}\right)\left(\mu_{2}-\mu_{1}\right) \mathrm{e}^{\mu_{2} l_{0}}}
\end{array}\right.
$$

\subsubsection{The critically damped case ( $\left.\beta_{0}=1\right)$}

In this case, when $\beta_{0}=1$, a pair of repeated real roots is obtained:

$$
\mu_{1}=\mu_{2}=-\alpha \beta_{0}
$$

According to the solution of $\mathrm{G}$ (s) with over damping conditions above, the expression of $\mathrm{G}(\mathrm{s})$ with the similar condition can be obtained as follows:

$$
\begin{aligned}
& G(s)=\left(C_{3}+C_{4} s\right) \mathrm{e}^{\mu_{1} s}+\mathrm{e}^{\mu_{1} s} \int_{s}^{l_{0}}\left[x \int_{s}^{l_{0}} R\left(\frac{2 c l_{0}}{v_{l}}-\frac{v_{r}}{v_{l}} x\right) \mathrm{e}^{-\mu_{1} x} \mathrm{~d} x\right] \mathrm{ds} \\
& =\left(C_{3}+C_{4} s\right) \mathrm{e}^{\mu_{1} s}+\mathrm{e}^{\mu_{1} s}\left[s \int_{s}^{l_{0}} R\left(\frac{2 c l_{0}}{v_{l}}-\frac{v_{r}}{v_{l}} x\right) \mathrm{e}^{-\mu_{1} x} \mathrm{~d} x-\int_{s}^{l_{0}} x R\left(\frac{2 c l_{0}}{v_{l}}-\frac{v_{r}}{v_{l}} x\right) \mathrm{e}^{-\mu_{1} x} \mathrm{dx}\right]
\end{aligned}
$$

where, from Eq. (26) and Eq. (33), the parameters of $C_{3}$ and $C_{4}$ are obtained by:

$$
\left\{\begin{array}{l}
C_{3}=\frac{v_{r} \phi\left(l_{0}\right)}{\left(v_{r}+v_{l}\right) \mathrm{e}^{\mu_{1} l_{0}}}-C_{4} l_{0} \\
C_{4}=\frac{v_{r} \phi\left(l_{0}\right)-\mu_{1} v_{r} \phi\left(l_{0}\right)+\psi\left(l_{0}\right)}{\left(v_{r}+v_{l}\right) \mathrm{e}^{\mu_{1} l_{0}}}
\end{array}\right.
$$

3.3.3 The underdamped case $\left(\beta_{0}<1\right)$

When $\beta_{0}<1$, the roots of Eq. (27) are the complex conjugate pair is given by:

$$
\left\{\begin{array}{l}
\mu_{1}=\alpha\left(-\beta_{0}+i \sqrt{1-\beta_{0}^{2}}\right) \\
\mu_{2}=\alpha\left(-\beta_{0}-i \sqrt{1-\beta_{0}^{2}}\right)
\end{array}\right.
$$

For convenience, introduce the parameters $\kappa=-\alpha \beta_{0}, \gamma=\alpha \sqrt{1-\beta_{0}^{2}}$, then $\mu_{1}$ and $\mu_{2}$ are expressed as:

$$
\left\{\begin{array}{l}
\mu_{1}=\kappa+i \gamma \\
\mu_{2}=\kappa-i \gamma
\end{array}\right.
$$

Hence, the expression of $\mathrm{G}(\mathrm{s})$ with under damped condition follows as: 


$$
\begin{aligned}
& G(s)=\mathrm{e}^{\kappa s}\left[C_{5} \cos (\gamma s)+C_{6} \sin (\gamma s)\right] \\
& +\frac{1}{\gamma} \mathrm{e}^{\kappa s}\left[\begin{array}{l}
\sin (\gamma s) \int_{s}^{l_{0}} R\left(\frac{2 c l_{0}}{v_{l}}-\frac{v_{r}}{v_{l}} x\right) \mathrm{e}^{-\kappa x} \cos (\gamma x) \mathrm{d} x- \\
\cos (\gamma s) \int_{s}^{l_{0}} R\left(\frac{2 c l_{0}}{v_{l}}-\frac{v_{r}}{v_{l}} x\right) \mathrm{e}^{-\kappa x} \sin (\gamma x) \mathrm{d} x
\end{array}\right]
\end{aligned}
$$

where,

$$
\left\{\begin{array}{l}
C_{5}=\frac{v_{r} \phi\left(l_{0}\right)}{\left(v_{r}+v_{l}\right) \mathrm{e}^{\kappa l_{0}} \cos \left(\gamma l_{0}\right)}-C_{6} \tan \left(\gamma l_{0}\right) \\
C_{6}=\frac{v_{r} \cos \left(\gamma l_{0}\right) \phi^{\prime}\left(l_{0}\right)+v_{r} \phi\left(l_{0}\right)\left[\gamma \sin \left(\gamma l_{0}\right)-\kappa \cos \left(\gamma l_{0}\right)\right]+\psi\left(l_{0}\right) \cos \left(\gamma l_{0}\right)}{\kappa \gamma\left(v_{r}+v_{l}\right) \mathrm{e}^{\kappa l_{0}}}
\end{array}\right.
$$

To summarize, combining Eqs.(21), (30), (33) and Eq. (36), one has the expressions for the boundary reflection with a fixed mass-spring-dashpot boundary condition:

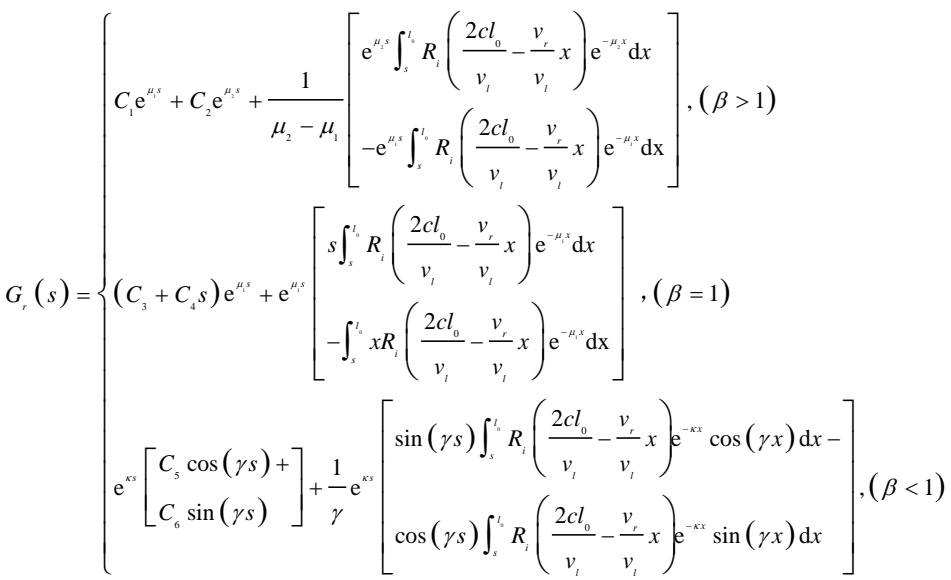

$$
\begin{aligned}
& F_{r}(s)=-G_{i}\left(-\frac{v_{l}}{v_{r}} s\right)
\end{aligned}
$$

where, $F_{i}, G_{i}$ represent the incident wave $(i=1,2,3, \ldots)$ and $F_{r}, G_{r}$ represent the reflection wave $(r=i+1)$, which are shown in Fig.2.

\section{The solution for the vibration response based on boundary reflection laws}

\subsection{The cycle of boundary reflection}

The cycle of motion of a travelling wave is defined as the minimum cycle time $T_{0}$ required to return to the initial deflection shape of a string with constant length after a 
series of boundary reflections which is given by [18]:

$$
T_{0}=\frac{l_{0}}{v_{r}}+\frac{l_{0}}{v_{l}}=\frac{2 c l_{0}}{c^{2}-v^{2}}
$$

where $v_{l}$ and $v_{r}$ are the wave propagation speeds to the left and to the right, respectively, according to the fixed coordinate system. So $T_{0}$ is divided into three time intervals: [0, $\left.t_{a}\right],\left[t_{a}, t_{b}\right]$ and $\left[t_{b}, T_{0}\right]$. The definitions and detailed expressions for $t_{a}$ and $t_{b}$ are shown in the reflected wave superposition method given in Ref [14]. The left-propagating waves $G_{i}$ and the right-propagating waves $F_{i}(i=1,2,3)$ during three time intervals are shown in Figure 2. The analytical solutions for the vibration responses are given next for these three time intervals for three kinds of non-classical boundary conditions.

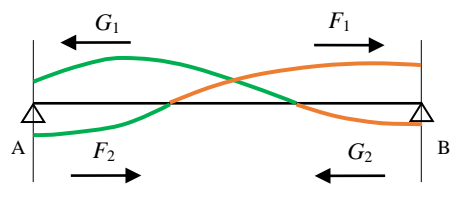

(a)

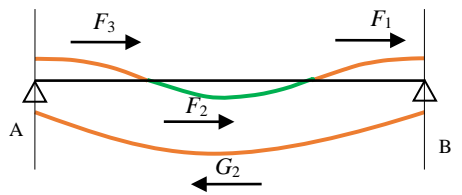

(c)

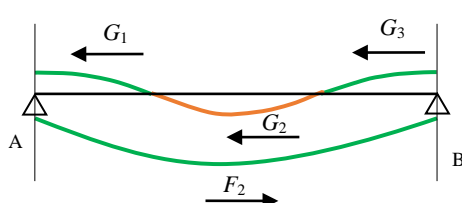

(b)

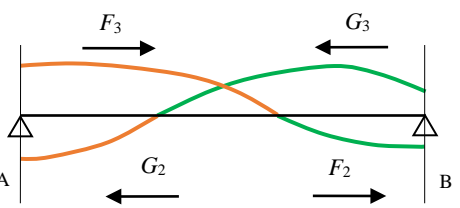

(d)

Fig.2 The propagating waves in a travelling string during three time intervals. (a) is for $\left[0, t_{a}\right]$, (b) is for $\left[t_{a}, t_{b}\right]$ when $v>0$, (c) is for $\left[t_{a}, t_{b}\right]$ when $v<0$ and (d) is for $\left[t_{b}, T_{0}\right] . F_{2}$ and $G_{2}$ are the reflection of waves $G_{1}$ and $F_{1} . F_{3}$, and $G_{3}$ are the reflection of waves $G_{2}$ and $F_{2}$.

\subsection{Fixed dashpot case}

In this case, according to the division of the boundary reflection process in a single cycle, the following three steps are taken to obtain the vibration response of the axial travelling string system.

\subsection{1 $0<t<t_{a}$}

According to Eq.(7), the expressions for initial travelling wave $F_{1}$ and $G_{1}$ are as follows:

$$
\left\{\begin{array}{l}
F_{1}\left(x-v_{r} t\right)=\frac{v_{l}}{v_{r}+v_{l}} \phi\left(x-v_{r} t\right)+\frac{1}{v_{r}+v_{l}} \int_{x-v_{r} t}^{l_{0}} \psi(\xi) \mathrm{d} \xi+C \\
G_{1}\left(x+v_{l} t\right)=\frac{v_{r}}{v_{r}+v_{l}} \phi\left(x+v_{l} t\right)-\frac{1}{v_{r}+v_{l}} \int_{x+v_{l} t}^{l_{0}} \psi(\xi) \mathrm{d} \xi-C
\end{array}\right.
$$

In this time interval as shown in Fig.2 (a), $G_{2}$ is the reflected wave of $F_{1}$ at the right-side boundary. Using the equation of boundary reflection Eq.(4) and the continuity 
condition, i.e. $G_{2}\left(l_{0}\right)=G_{1}\left(l_{0}\right)$, one has

$$
\begin{aligned}
G_{2}\left(x+v_{l} t\right)= & G_{1}\left(l_{0}\right)+\beta \frac{v_{l}}{v_{r}} F_{1}\left(l_{0}\right)-\beta \frac{v_{l}}{v_{r}} F_{1}\left(\frac{2 c}{v_{l}} l_{0}-\frac{v_{r}}{v_{l}} x-v_{r} t\right) \\
= & \frac{v_{r}^{2}+\beta v_{l}^{2}}{v_{r}\left(v_{r}+v_{l}\right)} \phi\left(l_{0}\right)-\frac{\beta v_{l}^{2}}{v_{r}\left(v_{r}+v_{l}\right)} \phi\left(\frac{2 c}{v_{l}} l_{0}-\frac{v_{r}}{v_{l}} x-v_{r} t\right) \\
& \left.-\frac{1}{v_{r}+v_{l}} \int_{\left(\frac{2 c}{l_{0}} l_{l} l_{0}-\frac{v_{r}}{v_{l}} x-v_{r} t\right.}\right) \psi(\xi) \mathrm{d} \xi+C
\end{aligned}
$$

$F_{2}$ is the reflected wave of $G_{1}$ at the left-side boundary. Using the equation for a boundary reflection Eq.(4), one has

$$
F_{2}\left(x-v_{r} t\right)=-\frac{v_{r}}{v_{r}+v_{l}} \phi\left(-\frac{v_{l}}{v_{r}}\left(x-v_{r} t\right)\right)+\frac{1}{v_{r}+v_{l}} \int_{-\frac{v_{l}}{v_{r}}\left(x-v_{r} t\right)}^{l_{0}} \psi(\xi) \mathrm{d} \xi+C
$$

Hence, one has the expressions:

$$
\begin{aligned}
& F\left(x-v_{r} t\right)= \begin{cases}F_{1}\left(x-v_{r} t\right), & v_{r} t<x<l_{0} \\
F_{2}\left(x-v_{r} t\right), & 0<x<v_{r} t\end{cases} \\
& G\left(x+v_{l} t\right)= \begin{cases}G_{1}\left(x+v_{l} t\right), & 0<x<l_{0}-v_{l} t \\
G_{2}\left(x+v_{l} t\right), & l_{0}-v_{l} t<x<l_{0}\end{cases}
\end{aligned}
$$

Finally, the expressions for the general solution of the equation of motion is

$$
u(x, t)=F\left(x-v_{r} t\right)+G\left(x+v_{l} t\right), 0<t<t_{a}
$$

4.2.2 $t_{a}<t<t_{b}$

When the string is translating to the right i.e. $v>0$, as shown in Fig. 2(b), one can see that the left end of $F_{1}$ disappears at the right boundary and $G_{3}$ is the reflected wave of $F_{2}$ at the right boundary. Using Eq.(12),one has

$$
G_{3}\left(x+v_{l} t\right)=G_{3}\left(l_{0}\right)+\beta \frac{v_{l}}{v_{r}} F_{2}\left(l_{0}\right)-\beta \frac{v_{l}}{v_{r}} F_{2}\left(\frac{2 c}{v_{l}} l_{0}-\frac{v_{r}}{v_{l}} x-v_{r} t\right)
$$

According to the continuity conditions, one can obtain:

$$
\begin{gathered}
G_{3}\left(l_{0}\right)=G_{2}\left(l_{0}+v_{l} t_{a}\right)=G_{2}\left(l_{0}\right)+\beta \frac{v_{l}}{v_{r}} F_{1}\left(l_{0}\right)-\beta \frac{v_{l}}{v_{r}} F_{1}\left(\frac{2 c}{v_{l}} l_{0}-\frac{v_{r}}{v_{l}} l_{0}-v_{r} t_{a}\right) \\
=\frac{v_{r}}{v_{r}+v_{l}} \phi\left(l_{0}\right)+\beta \frac{v_{l}}{v_{r}} \frac{v_{l}}{v_{r}+v_{l}} \phi\left(l_{0}\right)-\beta \frac{v_{l}}{v_{r}} \frac{v_{l}}{v_{r}+v_{l}} \phi(0) \\
F_{2}\left(l_{0}\right)=F_{2}\left(l_{0}-v_{r} t_{a}\right)=-\frac{v_{r}}{v_{r}+v_{l}} \phi(0)
\end{gathered}
$$

Substituting Eq. (47.a) and Eq. (47.b) into Eq. (47), the expression for the specific wave $G_{3}$ can be obtained as follows: 


$$
G_{3}\left(x+v_{l} t\right)=\frac{1}{v_{r}+v_{l}}\left(v_{r}+\beta \frac{v_{l}^{2}}{v_{r}}\right)\left[\phi\left(l_{0}\right)-\phi(0)\right]+\beta \frac{v_{l}}{v_{r}+v_{l}} \phi\left(x+v_{l} t-\frac{2 c}{v_{r}} l_{0}\right) \text { (47.c) }
$$

when $v<0$, one can see that the right end of $G_{1}$ disappears at the left boundary and $F_{3}$ is the reflected wave of $G_{2}$ at the right boundary. Using Eq.(12) and Eq.(42), one has

$$
F_{3}\left(x-v_{r} t\right)=-G_{2}\left(l_{0}\right)-\beta \frac{v_{l}}{v_{r}} F_{1}\left(l_{0}\right)+\beta \frac{v_{l}}{v_{r}} F_{1}\left(\frac{2 c}{v_{l}} l_{0}+x-v_{r} t\right)
$$

According to the continuity conditions, i.e. $G_{2}\left(l_{0}\right)=G_{2}\left(l_{0}+v_{l} t_{b}\right), F_{1}\left(l_{0}\right)=F_{1}\left(l_{0}-v_{r} t_{b}\right)$, one has

$$
\begin{array}{r}
G_{2}\left(l_{0}\right)=G_{2}\left(l_{0}\right)+\beta \frac{v_{l}}{v_{r}} F_{1}\left(l_{0}\right)-\beta \frac{v_{l}}{v_{r}} F_{1}\left(\frac{2 c}{v_{l}} l_{0}-\frac{v_{r}}{v_{l}} l_{0}-v_{r} t_{b}\right) \\
=\frac{v_{r}}{v_{r}+v_{l}} \phi\left(l_{0}\right)+\beta \frac{v_{l}}{v_{r}} \frac{v_{l}}{v_{r}+v_{l}} \phi\left(l_{0}\right)-\beta \frac{v_{l}}{v_{r}} \frac{v_{l}}{v_{r}+v_{l}} \phi\left(-\frac{2 v}{v_{l}} l_{0}\right) \\
F_{1}\left(l_{0}\right)=F_{1}\left(l_{0}-v_{r} t_{b}\right)=-\frac{v_{r}}{v_{r}+v_{l}} \phi\left(-\frac{2 v}{v_{l}} l_{0}\right)
\end{array}
$$

Substituting Eq. (48.a) and Eq. (48.b) into Eq. (48), the expression for $F_{3}$ can be obtained as follows:

$$
F_{3}\left(x-v_{r} t\right)=-\frac{1}{v_{r}+v_{l}}\left(v_{r}+\beta \frac{v_{l}^{2}}{v_{r}}\right) \phi\left(l_{0}\right)+\beta \frac{v_{l}}{v_{r}} \frac{v_{l}}{v_{r}+v_{l}} \phi\left(\frac{2 c}{v_{l}} l_{0}+x-v_{r} t\right)
$$

In total, one has the expressions:

$$
\begin{gathered}
F\left(x-v_{r} t\right)= \begin{cases}F_{1}\left(x-v_{r} t\right), & v_{r} t<x<l_{0}, v<0 \\
F_{2}\left(x-v_{r} t\right), & 0<x<l_{0}, v>0 \\
F_{2}\left(x-v_{r} t\right), & v_{r} t-\frac{v_{r}}{v_{l}} l_{0}<x<v_{r} t, v<0 \\
F_{3}\left(x-v_{r} t\right), & 0<x<v_{r} t-\frac{v_{r}}{v_{l}} l_{0}, v<0\end{cases} \\
G\left(x+v_{l} t\right)= \begin{cases}G_{1}\left(x+v_{l} t\right), & 0<x<l_{0}-v_{l} t, v>0 \\
G_{2}\left(x+v_{l} t\right), & l_{0}-v_{l} t<x<\frac{2 c l_{0}}{v_{r}}-v_{l} t, v>0 \\
G_{2}\left(x+v_{l} t\right), & 0<x<l_{0}, v<0 \\
G_{3}\left(x+v_{l} t\right), & \frac{2 c l_{0}}{v_{r}}-v_{l} t<x<l_{0}, v>0\end{cases}
\end{gathered}
$$

Finally, the expressions of the general solution of the equation of motion is

$$
u(x, t)=F\left(x-v_{r} t\right)+G\left(x+v_{l} t\right), t_{a}<t<t_{b}
$$

4.2.3 $t_{b}<t<T_{0}$

In this time interval, as shown in Fig. 2(d), the expressions for the propagating 
waves $F_{2}, F_{3}, G_{2}$ and $G_{3}$ are the same as the previous ones except for the different ranges of values of $x$. One has the expressions:

$$
\begin{gathered}
F\left(x-v_{r} t\right)=\left\{\begin{array}{l}
F_{2}\left(x-v_{r} t\right), v_{r} t-\frac{v_{r}}{v_{l}} l_{0}<x<l_{0} \\
F_{3}\left(x-v_{r} t\right), 0<x<v_{r} t-\frac{v_{r}}{v_{l}} l_{0}
\end{array}\right. \\
G\left(x+v_{l} t\right)=\left\{\begin{array}{l}
G_{2}\left(x+v_{l} t\right), 0<x<\frac{2 c l_{0}}{v_{r}}-v_{l} t \\
G_{3}\left(x+v_{l} t\right), \frac{2 c l_{0}}{v_{r}}-v_{l} t<x<l_{0}
\end{array}\right.
\end{gathered}
$$

Finally, the expressions for the general solution of the equation of motion is

$$
u(x, t)=F\left(x-v_{r} t\right)+G\left(x+v_{l} t\right), t_{b}<t<T_{0}
$$

Taking into account the continuity and the first order smoothness, the initial out of plane string velocity and displacement conditions are chosen to be

$$
\left\{\begin{array}{l}
\psi(x)=0 \\
\phi(x)=\left[H(x)-H\left(x-l_{0}\right)\right] A_{0}\left(\sin \frac{\pi x}{l_{0}}\right)^{2}
\end{array}\right.
$$

where, $H$ is the Heaviside step function and $A_{0}$ is amplitude of incident wave.

The dimensionless displacement at a point on the travelling string using the proposed wave superposition method are compared with numerical solutions of an FE model solved using the Newmark- $\beta$ method[12], which are shown in Fig. 3. The parameters for the axial travelling string system are selected as: an initial amplitude $A_{0}=$ $0.01 \mathrm{~m}$, the length of string is $l_{0}=1 \mathrm{~m}$, the linear damping coefficient is $\eta=0.5 \mathrm{Ns} / \mathrm{m}$ and the dimensionless translational speed of the string is $V=v / c$. 

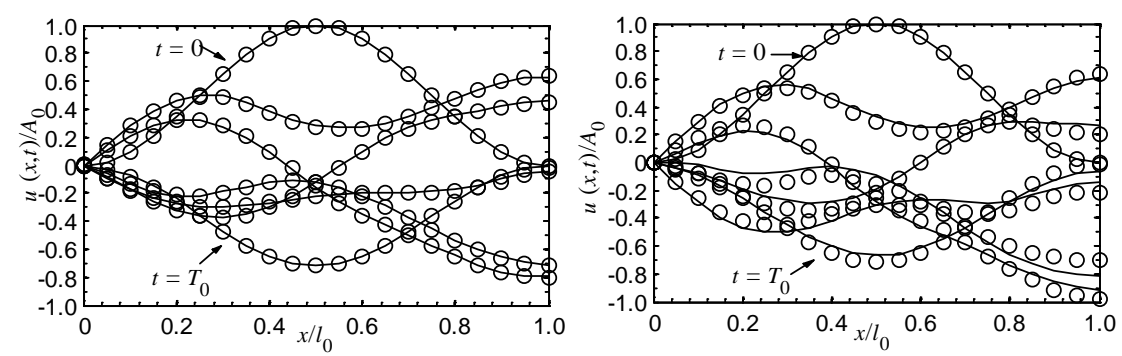

(a)

(b)

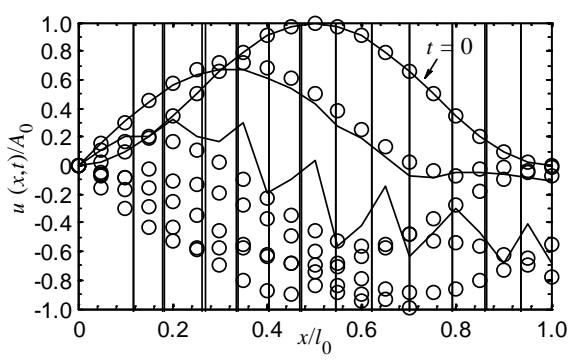

(c)

Fig.3 Dimensionless travelling string free vibration transverse displacement response with a fixed dashpot boundary at the dimensionless travelling speeds of (a) $V=0.1$, (b) $V=0.2$ and (c) $V=0.9$. The curves are identified by: $\circ \circ \circ$ wave superposition method; - - Newmark- $\beta$ method.

The proposed method is well in agreement with the Newmark- $\beta$ method solution of a FE model at low travelling speed, while there are increasing differences as the travelling speed increases. When the travelling speed is close to the critical speed $c$ in Fig.3(c), the proposed method is stable while the Newmark- $\beta$ method has begun to diverge. It can be seen clearly in Fig.3(a) that the string vibrates from the top to the bottom and the phase of all points on the string change by $\pi$ in the time interval of $\left[0, T_{0}\right]$. The amplitude of all points at time $T_{0}$ is different from the initial ones due to the exchange of energy at the boundaries.

\subsection{Fixed spring-dashpot case}

Similarly, according to the reflection relationships at the fixed and spring-dashpot boundaries given in Section 3.2, the expressions for the propagating waves in the corresponding three time intervals $\left[0, t_{a}\right],\left[t_{a}, t_{b}\right]$ and $\left[t_{b}, T_{0}\right]$ can be obtained as follows

$$
F_{2}\left(x-v_{r} t\right)=-\frac{v_{r}}{v_{r}+v_{l}} \phi\left(v_{l} t-\frac{v_{l}}{v_{r}} x\right)+\frac{1}{v_{r}+v_{l}} \int_{v_{t} t-\frac{v_{1}}{v_{r}} x}^{l_{0}} \psi(\xi) \mathrm{d} \xi+C
$$




$$
\begin{gathered}
G_{2}\left(x+v_{l} t\right)=\left(G_{2}\left(l_{0}\right)+\beta \frac{v_{l}}{v_{r}} F_{1}\left(l_{0}\right)\right) \mathrm{e}^{\alpha\left(x+v_{l} t-l_{0}\right)}-\beta \frac{v_{l}}{v_{r}} F_{1}\left(\frac{2 c}{v_{l}} l_{0}-\frac{v_{r}}{v_{l}} x-v_{r} t\right)+ \\
\alpha\left(\beta \frac{v_{l}}{v_{r}}-1\right) \int_{x+v_{l} t}^{l_{l}} F_{1}\left(\frac{2 c}{v_{l}} l_{0}-\frac{v_{r}}{v_{l}} s\right) \mathrm{e}^{\alpha\left(x+v_{l} t-s\right)} \mathrm{d} s \\
F_{3}\left(x-v_{r} t\right)=-\left(G_{2}\left(l_{0}\right)+\beta \frac{v_{l}}{v_{r}} F_{1}\left(l_{0}\right)\right) \mathrm{e}^{\alpha\left(v_{l}-\frac{v_{l}}{v_{r}} x-l_{0}\right)}+\beta \frac{v_{l}}{v_{r}} F_{1}\left(\frac{2 c}{v_{l}} l_{0}+x-v_{r} t\right)- \\
\alpha\left(\beta \frac{v_{l}}{v_{r}}-1\right) \int_{v_{l} t-\frac{v_{l}}{v_{r}}}^{l_{0}} F_{1}\left(\frac{2 c}{v_{l}} l_{0}-\frac{v_{r}}{v_{l}} s\right) \mathrm{e}^{\alpha\left(v_{l} t-\frac{v_{l}}{v_{r}} x-s\right)} \mathrm{d} s \\
G_{3}\left(x+v_{l} t\right)=\left(G_{3}\left(l_{0}\right)+\beta \frac{v_{l}}{v_{r}} F_{2}\left(l_{0}\right)\right) \mathrm{e}^{\alpha\left(x+v_{l} t-l_{0}\right)}-\beta \frac{v_{l}}{v_{r}} F_{2}\left(\frac{2 c}{v_{l}} l_{0}-\frac{v_{r}}{v_{l}} x-v_{r} t\right)+ \\
\alpha\left(\beta \frac{v_{l}}{v_{r}}-1\right) \int_{x+v_{l} t}^{l_{l}} F_{2}\left(\frac{2 c}{v_{l}} l_{0}-\frac{v_{r}}{v_{l}} s\right) \mathrm{e}^{\alpha\left(x+v_{l} t-s\right)} \mathrm{d} s
\end{gathered}
$$

where, the expressions for $G_{1}$ and $F_{1}$ are identical to Eq. (41). Replacing $G_{2}, F_{2}, G_{3}, F_{3}$ in Eq. (42), Eq. (43), Eq. (47.c) and Eq. (48) with the corresponding ones in Eqs.(56) (59), respectively, one can obtain the analytical expressions for transverse displacement during the three time intervals in this case. At the same time, in order to satisfy the continuity condition $[14,21]$ at the boundaries, i.e.

$$
\left\{\begin{array}{l}
\left(1-v^{2}\right) \phi^{\prime}\left(l_{0}\right)+k \phi\left(l_{0}\right)=(v-\eta) \psi\left(l_{0}\right) \\
(v-\eta)\left(1-v^{2}\right) \phi^{\prime \prime}\left(l_{0}\right)=\left[1-v^{2}+2 v(v-\eta) \psi^{\prime}\left(l_{0}\right)\right]+k \psi\left(l_{0}\right)
\end{array}\right.
$$

The initial velocity and displacement are chosen as follows

$$
\left\{\begin{array}{l}
\psi(x)=0 \\
\phi(x)=\left[H(x)-H\left(x-l_{0}\right)\right] A_{0}\left(\sin \frac{\pi x}{l_{0}}\right)^{2}\left(\sin \frac{\pi\left(x-l_{0}\right)}{l_{0}}\right)^{2}
\end{array}\right.
$$

Fig. 4 shows the dimensionless string displacement for this case, whose parameters are identical to those in section 4.2 except for the additional stiffness of $k=0.5 \mathrm{~N} / \mathrm{m}$. It is clear in Fig.4(c) that, the Newmark- $\beta$ method is very divergent compared with the proposed method. 

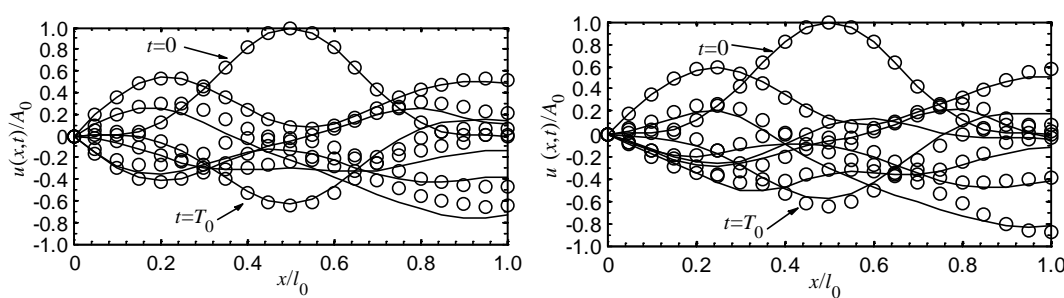

(a)

(b)

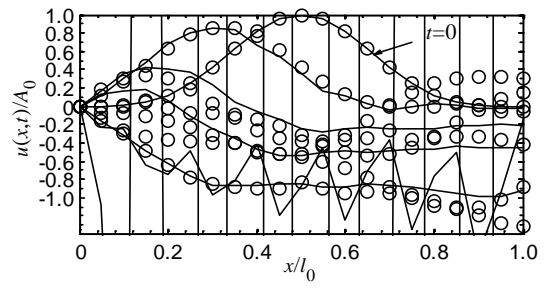

(c)

Fig.4 Displacement response of free transverse vibration with fixed spring-dashpot boundary at the dimensionless travelling speeds of (a) $V=0.1$, (b) $V=0.2$ and (c) $V=0.9$.The curves are identified by: $० \circ \circ$ wave superposition method; --- Newmark- $\beta$ method.

\subsection{Fixed mass-spring-dashpot case}

In this case, the expressions for $G_{1}$ and $F_{1}$ are identical to Eq. (41). According to the reflection equations Eqs. (38-39) for the fixed mass-spring-dashpot boundaries, one has the detailed expressions for the moving waves of $F_{2}, G_{2}, F_{3}, G_{3}$ during the three time intervals:

$$
F_{2}\left(x-v_{r} t\right)=-\frac{v_{r}}{v_{r}+v_{l}} \phi\left(\frac{v_{l}}{v_{r}}\left(v_{r} t-x\right)\right)+\frac{1}{v_{r}+v_{l}} \int_{\frac{v_{l}}{v_{r}}\left(v_{r} t-x\right)}^{l_{0}} \psi(\xi) \mathrm{d} \xi+C
$$




$$
\begin{aligned}
& G_{2}\left(x+v_{l} t\right)= \\
& {\left[C_{1} \mathrm{e}^{\mu_{1}\left(x+v_{l} t\right)}+C_{2} \mathrm{e}^{\mu_{2}\left(x+v_{l} t\right)}+\frac{1}{\mu_{2}-\mu_{1}}\left[\begin{array}{c}
\mathrm{e}^{\mu_{2}\left(x+v_{l} t\right)} \int_{x+v_{l} t}^{l_{0}} R_{1}\left(\frac{2 c l_{0}}{v_{l}}-\frac{v_{r}}{v_{l}} x\right) \mathrm{e}^{-\mu_{2} x} \mathrm{~d} x- \\
\mathrm{e}^{\mu_{1}\left(x+v_{l} t\right)} \int_{x+v_{l} t}^{l_{0}} R_{1}\left(\frac{2 c l_{0}}{v_{l}}-\frac{v_{r}}{v_{l}} x\right) \mathrm{e}^{-\mu_{1} x} \mathrm{~d} x
\end{array}\right], \quad(\beta>1)\right.} \\
& \left\{\left[C_{3}+C_{4}\left(x+v_{l} t\right)\right] \mathrm{e}^{\mu_{1}\left(x+v_{l} t\right)}+\mathrm{e}^{\mu_{1}\left(x+v_{l} t\right)}\left[\begin{array}{l}
\left(x+v_{l} t\right) \int_{x+v_{l} t}^{l_{0}} R_{1}\left(\frac{2 c l_{0}}{v_{l}}-\frac{v_{r}}{v_{l}} x\right) \mathrm{e}^{-\mu_{1} x} \mathrm{~d} x- \\
\int_{x+v_{l}}^{l_{0}} x R_{1}\left(\frac{2 c l_{0}}{v_{l}}-\frac{v_{r}}{v_{l}} x\right) \mathrm{e}^{-\mu_{1} x} \mathrm{~d} x
\end{array}\right],(\beta=1)\right. \\
& \mathrm{e}^{\kappa\left(x+v_{l} t\right)}\left\{\left[\begin{array}{l}
\left.C_{5} \cos \left[\gamma\left(x+v_{l} t\right)\right]+\right] \\
C_{6} \sin \left[\gamma\left(x+v_{l} t\right)\right]
\end{array}\right]+\frac{1}{\gamma}\left[\begin{array}{l}
\sin \left[\gamma\left(x+v_{l} t\right)\right] \int_{x+v_{l} t}^{l_{0}} R_{1}\left(\frac{2 c l_{0}}{v_{l}}-\frac{v_{r}}{v_{l}} x\right) \mathrm{e}^{-\kappa x} \cos (\gamma x) \mathrm{d} x- \\
\cos \left(\gamma\left(x+v_{l} t\right)\right) \int_{x+v_{l} t}^{l_{0}} R_{1}\left(\frac{2 c l_{0}}{v_{l}}-\frac{v_{r}}{v_{l}} x\right) \mathrm{e}^{-\kappa x} \sin (\gamma x) \mathrm{d} x
\end{array}\right]\right\}, \\
& (\beta<1) \\
& \text { (63) } \\
& G_{3}\left(x+v_{l} t\right)=
\end{aligned}
$$

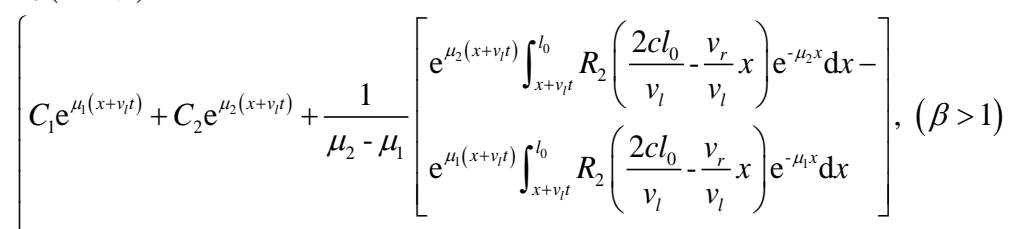

$$
\begin{aligned}
& \left\{\left[C_{3}+C_{4}\left(x+v_{l} t\right)\right] \mathrm{e}^{\mu_{1}\left(x+v_{l} t\right)}+\mathrm{e}^{\mu_{1}\left(x+v_{l} t\right)}\left[\begin{array}{l}
x \int_{x+v_{l} t}^{l_{0}} R_{2}\left(\frac{2 c l_{0}}{v_{l}}-\frac{v_{r}}{v_{l}} x\right) \mathrm{e}^{-\mu_{1} x} \mathrm{~d} x- \\
\int_{x+v_{l} t}^{l_{0}} x R_{2}\left(\frac{2 c l_{0}}{v_{l}}-\frac{v_{r}}{v_{l}} x\right) \mathrm{e}^{-\mu_{1} x} \mathrm{~d} x
\end{array}\right],(\beta=1)\right.
\end{aligned}
$$

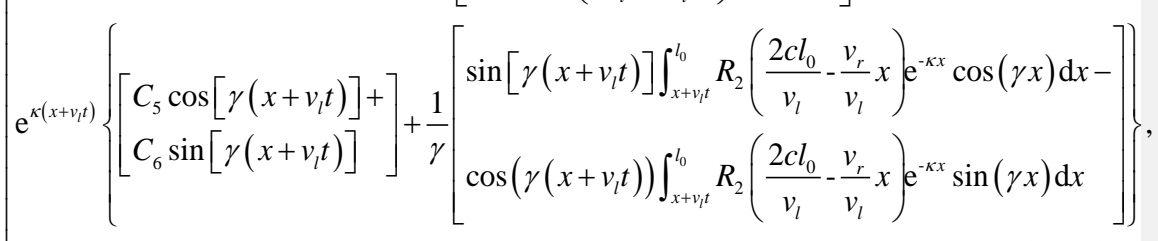

$$
\begin{aligned}
& (\beta<1)
\end{aligned}
$$




$$
\begin{aligned}
& F_{3}\left(x-v_{r} t\right)=
\end{aligned}
$$

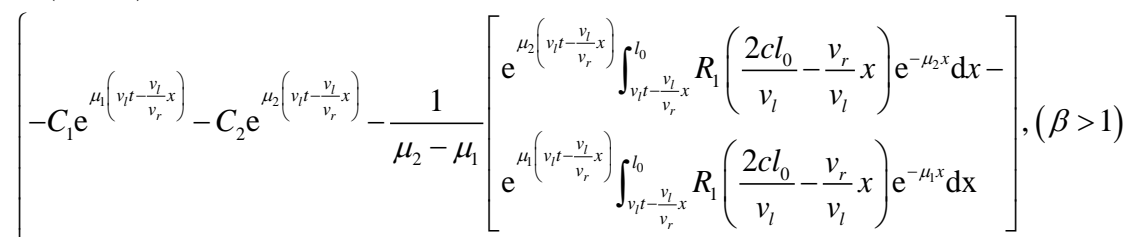

$$
\begin{aligned}
& -\left[C_{3}+C_{4}\left(v_{l} t-\frac{v_{l}}{v_{r}} x\right)\right] \mathrm{e}^{\mu_{1}\left(v_{l} t-\frac{v_{l}}{v_{r}} x\right)}-\mathrm{e}^{\mu_{l}\left(v_{l} t-\frac{v_{l}}{v_{r}} x\right)}\left[\begin{array}{l}
\left(\begin{array}{l}
v_{l} t-\frac{v_{l}}{v_{r}} x \\
v_{r}
\end{array}\right) \int_{v_{l} t-\frac{v_{v}}{v_{r}} x}^{l_{1}} R_{1}\left(\frac{2 c l_{0}}{v_{l}}-\frac{v_{r}}{v_{l}} x\right) \mathrm{e}^{-\mu_{1} x} \mathrm{~d} x- \\
\int_{v_{l} t-\frac{v_{l}}{v_{r}} x}^{l_{1}} x R_{1}\left(\frac{2 c l_{0}}{v_{l}}-\frac{v_{r}}{v_{l}} x\right) \mathrm{e}^{-\mu_{1} x} \mathrm{dx}
\end{array}\right], \\
& (\beta=1)
\end{aligned}
$$

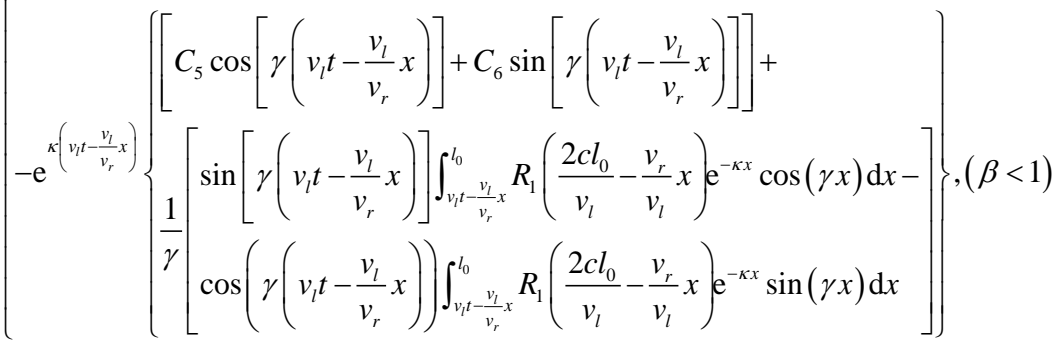

where,

$$
\begin{aligned}
R_{1}\left(\frac{2 c l_{0}}{v_{l}}-\frac{v_{r}}{v_{l}} X\right)= & -\frac{v_{r}^{2}}{v_{l}^{2}} F_{1}^{\prime \prime}\left(\frac{2 c l_{0}}{v_{l}}-\frac{v_{r}}{v_{l}} x\right)+\frac{\eta v_{r}-\rho v c-T}{m v_{l}^{2}} F_{1}^{\prime}\left(\frac{2 c l_{0}}{v_{l}}-\frac{v_{r}}{v_{l}} X\right)- \\
& \frac{k}{m v_{l}^{2}} F_{1}\left(\frac{2 c l_{0}}{v_{l}}-\frac{v_{r}}{v_{l}} x\right) \\
R_{2}\left(\frac{2 c l_{0}}{v_{l}}-\frac{v_{r}}{v_{l}} X\right)= & -\frac{v_{r}^{2}}{v_{l}^{2}} F_{2}^{\prime \prime}\left(\frac{2 c l_{0}}{v_{l}}-\frac{v_{r}}{v_{l}} X\right)+\frac{\eta v_{r}-\rho v c-T}{m v_{l}^{2}} F_{2}^{\prime}\left(\frac{2 c l_{0}}{v_{l}}-\frac{v_{r}}{v_{l}} X\right)- \\
& \frac{k}{m v_{l}^{2}} F_{2}\left(\frac{2 c l_{0}}{v_{l}}-\frac{v_{r}}{v_{l}} x\right)
\end{aligned}
$$

Replacing $G_{2}, F_{2}, G_{3}, F_{3}$ in Eq. (42), Eq.(43), Eq.(47.c) and Eq.(48) with the corresponding ones in Eqs.(62) - (65), respectively, one can obtain the analytical transverse displacement for the travelling string during three time intervals in the fixed mass-spring-dashpot case. In order to satisfy the boundary continuity condition of Eq.(4), the initial conditions for the velocity and displacement are given as follows: 


$$
\left\{\begin{array}{l}
\psi(x)=0 \\
\phi(x)=\left[\mathrm{H}(x)-H\left(x-l_{0}\right)\right] A_{0}\left(\sin \frac{\pi x}{l_{0}}\right)^{3}\left(\sin \frac{\pi\left(x-l_{0}\right)}{l_{0}}\right)^{3}
\end{array}\right.
$$

Fig. 5 shows the dimensionless string displacement for this case whose parameters are identical to those in section 4.3 except for the attached mass of $m=0.01 \mathrm{~kg}$. As the initial conditions in Eq.(68) for this case are different from the first two cases, the vibration for the string during the time interval of $\left[0, T_{0}\right]$ starts at the bottom and ends at the top.

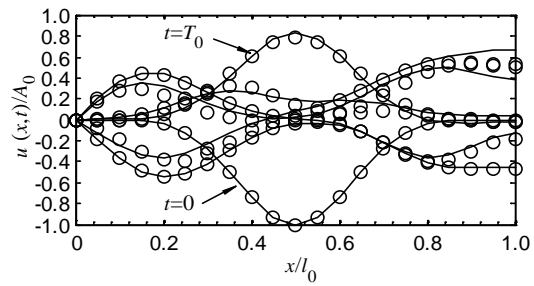

(a)

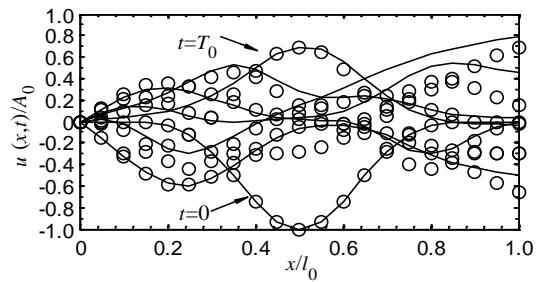

(b)

Fig.5 Displacement response of free transverse vibration with a fixed mass-spring-dashpot boundary at the dimensionless travelling speeds of (a) $V=0.1$ and (b) $V=0.2$. The curves are identified by: ००० wave superposition method; --- Newmark- $\beta$ method.

\section{Vibrational energy analysis}

In this section, the fixed dashpot boundary condition is taken as an example to obtain vibrational energy based on the proposed reflected wave superposition method. The relationship between energy and velocity of string is also discussed.

The total mechanical energy $E(t)$ of the axially travelling string comprises the kinetic and potential energy in the following way [6]:

$$
E(t)=\frac{\rho}{2} \int_{0}^{l(t)}\left[\left(u_{t}+v u_{x}\right)^{2}+c^{2} u_{x}^{2}\right] \mathrm{d} x
$$

From Eq. (5), we obtain Eq. (70)

$$
\left\{\begin{array}{l}
u_{t}=-v_{r} F^{\prime}+v_{l} G^{\prime} \\
u_{x}=F^{\prime}+G^{\prime}
\end{array}\right.
$$

Incorporating the Eq. (70) into Eq. (69), one has:

$$
\begin{aligned}
E(t) & =\frac{\rho}{2} \int_{0}^{l(t)}\left[\left(-v_{r} F^{\prime}+v_{l} G^{\prime}+v F^{\prime}+v G^{\prime}\right)^{2}+c^{2}\left(F^{\prime}+G^{\prime}\right)^{2}\right] \mathrm{d} x \\
& =\rho c^{2} \int_{0}^{l(t)}\left(F^{\prime 2}+G^{\prime 2}\right) \mathrm{d} x
\end{aligned}
$$

In Ref.[14], the energy calculation method for an axially travelling string with 
fixed-fixed boundary condition is given analytically. Here, the energy expressions for a travelling string with a fixed dashpot boundary condition can be obtained simply by using the new formulae for $G_{2}, G_{3}$ and $F_{3}$ given in Eqs.(42), (47.c) and (48.c) to replace the corresponding functions in the energy calculation method proposed in [14].

Fig.6 shows the curves for the total energy for a travelling string $(V=0.5)$ with fixed dashpot boundary conditions and different levels of viscous damping for the first five response cycles. When $\eta=0 \mathrm{Ns} / \mathrm{m}$, the values of energy are equal at the beginning and end of the cycle, but fluctuate during the cycle. The energy curves of subsequent cycles are identical as that of the first cycle. These indicate that the energy gained by the travelling string at the downstream boundary at an instantaneous moment is not equal to the energy lost at the upstream boundary, but the total energy gained during a cycle is equal to the total energy lost. When $\eta>0$, the energy of the travelling string system decreases in every cycle, and the larger the viscous damping the more rapid the attenuation.

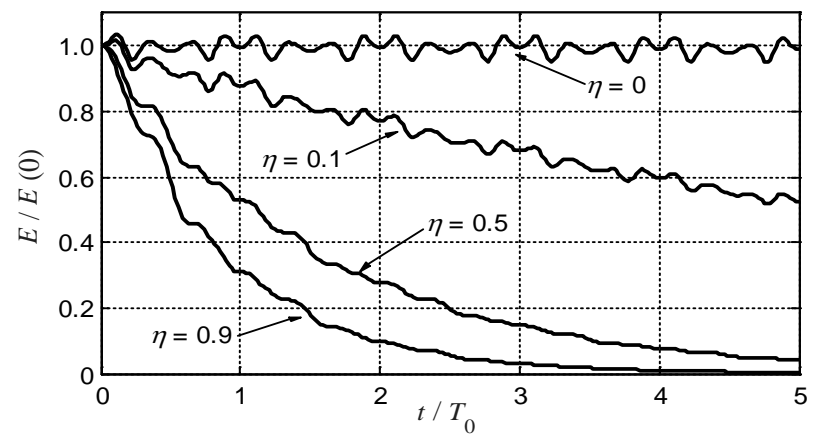

Fig. 6 The change in the transverse free vibration energy of an axially travelling string for fixed dashpot boundary conditions with viscous damping constant $\eta=0 \mathrm{Ns} / \mathrm{m}, 0.1 \mathrm{Ns} / \mathrm{m}, 0.5 \mathrm{Ns} / \mathrm{m}$ and 0.9 $\mathrm{Ns} / \mathrm{m}$ during the first five cycles. 

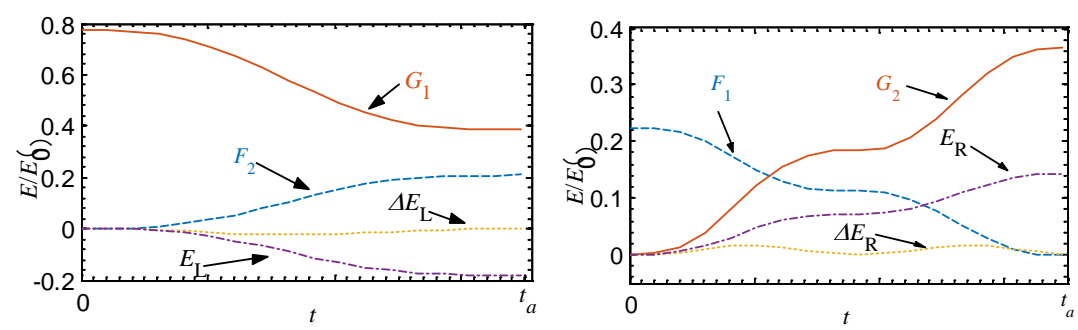

(a)

(b)
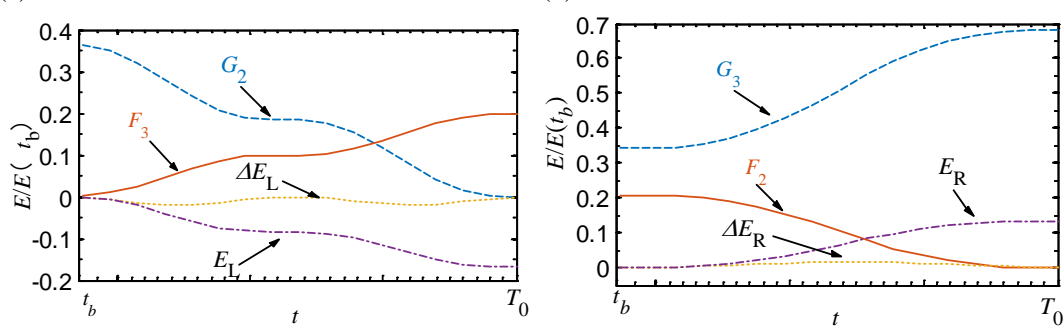

(c)

(d)

Fig.7 Variations in the energy of the propagating waves $\left(F_{1}, F_{2}, F_{3}\right.$ and $\left.G_{1}, G_{2}, G_{3}\right)$ in a travelling string $(V=0.5)$ with viscous damping of $\eta=0.1 \mathrm{Ns} / \mathrm{m}$ at the boundaries. The effect due to their reflections at boundaries of (a) $x=0$ during time interval $\left[0, t_{a}\right]$, (b) $x=l_{0}$ during time interval $\left[0, t_{a}\right]$, (c) $x=0$ during time interval $\left[t_{b}, T_{0}\right]$ and (d) $x=l_{0}$ during time interval $\left[t_{b}, T_{0}\right] . E_{\mathrm{L}}$ and $E_{\mathrm{R}}$ are the variations of energy for the travelling string system due to the left boundary $(x=0)$ and the right boundary $\left(x=l_{0}\right)$ respectively from 0 to $t$ at (a) and (b) or from $t_{b}$ to $t$ at (c) and (d). $\Delta E_{\mathrm{L}}$ and $\Delta E_{\mathrm{R}}$ are the change in the energy due to the left boundary $(x=0)$ and the right boundary $\left(x=l_{0}\right)$ respectively, $\Delta E_{\mathrm{L}}(i)=E_{\mathrm{L}}(i)-E_{\mathrm{L}}(i-1)$ and $\Delta E_{\mathrm{R}}(i)=E_{\mathrm{R}}(i)-E_{\mathrm{R}}(i-1)$.

Fig.7(a) shows the reflection at the fixed boundary of $x=0$ during time interval $\left[0, t_{a}\right]$ with the incident wave $G_{1}$ and reflected wave $F_{2}$. The energy of $G_{1}$ decreases and the energy of $F_{2}$ increases. The change in the energy $E_{\mathrm{L}}$ for the travelling string system due to the left boundary $(x=0)$ is less than zero, which indicates that the energy flows out of the system from the left boundary. In Fig.7(b), $E_{\mathrm{L}}$ is greater than zero, which indicates that the energy flows into the system from the right boundary. However, the energy gained at the right boundary is less than the energy lost at the left boundary, e.g. $E_{\mathrm{L}}\left(t_{a}\right) \approx-0.18$ and $E_{\mathrm{R}}\left(t_{a}\right) \approx 0.14$. This is due to the viscous damper at the right boundary dissipating energy, so the overall energy of the system is reduced, as shown in Fig.6. From $\Delta E_{\mathrm{L}}$ and $\Delta E_{\mathrm{R}}$, one can judge whether the energy is flowing into $\left(\Delta E_{\mathrm{L}}\right.$ or $\left.\Delta E_{\mathrm{R}}>0\right)$ or out of $\left(\Delta E_{\mathrm{L}}\right.$ or $\left.\Delta E_{\mathrm{R}}<0\right)$ the system from the respective boundaries.

\section{Conclusions}

Based on Hamilton's principle and the Lagrangian function, three types of model of an axially travelling string with mixed boundary conditions are derived and the boundary equations for the axially moving string model are also obtained. 
The general one-dimensional wave solution of equation of motion for the string displacement is obtained using the D'Alembert method. Combining the general solution of the equation of motion and the boundary conditions, the boundary reflection law for different boundaries was derived.

A propagating and reflected wave superposition method is then proposed in this paper to obtain the analytical solutions for the free vibration of an axially translating string with a mixed boundary support. From application of this method, one can consider and examine the physical nature of the string vibration based on the concept of wave propagation. The reflected wave superposition method is very consistent with numerical solution obtained using the Newmark- $\beta$ method by comparing the displacement response, which verifies the accuracy of the proposed method.

Finally, we take the fixed dashpot boundary condition as an example to obtain vibrational energy based on the proposed reflected wave superposition method. The quantitative relationship between energy and velocity of string is discussed.

\section{Acknowledgments}

This work was supported by the National Natural Science Foundation of China [grant numbers 51675150 and 51305115].

\section{References}

[1] K.F. Graff, Wave Motion in Elastic Solids, Publication of Oxford University Press, London, 1975.

[2] W.A. Strauss, Partial Differential Equations: An Introduction, Wiley, New York, 1992.

[3] T. Akkaya, W.T. van Horssen, Reflection and damping properties for semi-infinite string equations with non-classical boundary conditions, J. Sound Vib. 336(2015) 179-190.

[4] N.V. Gaiko, W.T. van Horssen, On wave reflections and energetics for a semi-infinite travelling string with a nonclassical boundary support, J. Sound Vib. 370 (2016) 336-350.

[5] B. Yang, C.A. Tan, Transfer functions of one-dimensional distributed parameter systems, J. Appl. Mech. 59 (4) (1992) 1009-1014.

[6] C.A. Tan, S. Ying, Dynamic analysis of the axially moving string based on wave propagation, J. Appl. Mech. 64 (2) (1997) 394-400.

[7] W.T. van Horssen, On the influence of lateral vibrations of supports for an axially moving string, J. Sound Vib. 268 (2) (2003) 323-330.

[8] W.L. Miranker, The Wave Equation in a Medium in Motion[M], IBM Corp, 4 (1) (1960) 36-42.

[9] S.Y. Lee, M. Lee, A new wave technique for free vibration of a string with time-varying length, J. Appl. Mech. 69 (1) (2002) 83-87.

[10] W.T. van Horssen, On the weakly damped vibrations of a string attached to a spring mass dashpot system, J. Vib. Control 9 (11) (2003) 1231-1248.

[11] D. Darmawijoyo, The vibrations of a linear and a weakly 1-D wave equations with non-classical boundary damping, J. Appl. Sci. 11 (7) (2011) 1206-1212.
Commented [FN3]: Not sure whether you have added any comment regarding teh amount of energy added or removed as a function of the tranlation speed or level of the damper. Also, is it true that with dashpots at both ends then knwoing teh direction of translation one knows which end will add and which end will remove energy (by doing postive or negative work on the sysytem). I assume, but don't know, that the effect of addition and removal of energy is different than what happens at fixed boundaries and don't know whether it is greater or less.

As a possible thing to investigate in future work, is whether the mass-spring-damper being a tuned sysytem shows particular different behaviour compared to either the spring or spring-damper configuration? 
[12] E.W. Chen, N.S. Ferguson, Analysis of energy dissipation in an elastic moving string with a viscous damper at one end, J. Sound Vib. 333 (9) (2014) 2556-2570.

[13] N.V. Gaiko, W.T. van Horssen, On the transverse, low frequency vibrations of a traveling string with boundary damping, J. Vib. Acoust. 137 (4) (2015) 1316-1326.

[14] E.W. Chen, Q. Luo, N.S. Ferguson, A reflected wave superposition method for vibration and energy of a travelling string, J. Sound Vib. 400 (2017) 40-57.

[15] W. K. Chang, K. S. Hong, H. Park, Boundary control of an axially traveling string: Actuator dynamics included, J. Mech. Sci. Technol, 19(1) (2005)40-50.

[16] Q. H. Ngo, K. S. Hong, I. H. Jung, Adaptive control of an axially moving system, J. Mech. Sci. Technol, 23(11) (2009)3071-3078.

[17] W.T. van Horssen, Y. D. Wang, G.H. Cao, On solving wave equations on fixed bounded intervals involving Robin boundary conditions with time-dependent coefficients. J. Sound Vib. 424(2018) 263-271.

[18] S.Y. Lee, C.D. Mote Jr., A generalized treatment of the energetics of translating continua, Part I: strings and second order tensioned pipes, J. Sound Vib. 204 (5) (1997) 717-734.

[19] R.A. Sack, Transverse oscillations in travelling strings, Br. J. Appl. Phys. 5 (6) (1954) 224-226.

[20] D.B. McIver, Hamilton's principle for systems of changing mass, Journal of Engineering Mathematics 7(3) (1973)249-261.

[21] R.D. Swope, W.F. Ames, Vibrations of a moving thread line, J. Frankl. Inst. 275 (1) (1963) $36-55$. 